Chapter X

Mindfulness for Cultivating Self-Esteem

Christopher A. Peppinga*, Penelope J. Davis ${ }^{\mathrm{b}}$, and Analise O’Donovan ${ }^{\mathrm{b}}$

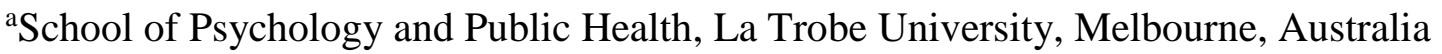

${ }^{\mathrm{b}}$ School of Applied Psychology, Behavioral Basis of Health, and Griffith Health Institute, Griffith University, Brisbane, Australia

Christopher A. Pepping $\square$

School of Psychology and Public Health, La Trobe University, Melbourne, Australia. Email: c.pepping@latrobe.edu.au

E. Y. Shonin, W. Van Gordon and M. D. Griffiths (eds.), Mindfulness and other BuddhistDerived Approaches in Mental Health and Addiction, Advances in Mental Health and Addiction Series. DOI: 
Abstract (to be included only in the e-Book version)

Self-esteem is said to be a fundamental and pervasive human need. Humans are motivated to maintain high self-esteem, and engage in efforts to protect their self-esteem when it comes under threat. High self-esteem is a positive personal resource, and is generally related to a wide range of positive life outcomes. It is therefore important to consider factors that may enhance self-esteem. Here we argue that mindfulness may be useful to cultivate self-esteem and, in particular, secure self-esteem as opposed to fragile high self-esteem. We begin with an overview of the benefits of healthy self-esteem and costs of low self-esteem. We then argue that individuals high in dispositional mindfulness may have greater capacity for secure high self-esteem. Evidence from clinical and experimental studies examining associations between mindfulness and self-esteem is reviewed next. We conclude with a discussion of the use of mindfulness-based interventions for the cultivation of healthy self-esteem and an outline of directions for future research.

Keywords: Mindfulness; Meditation; Self-esteem; Self-worth; Secure Self-esteem. 


\section{Mindfulness for Cultivating Self-Esteem}

Self-esteem is widely conceptualised as a fundamental and pervasive human need (Greenberg et al., 1992; Pyszczynski et al., 2004). In the present chapter we argue that mindfulness may be associated with increased self-esteem and, in particular, with secure forms of self-esteem rather than fragile high self-esteem. We begin by providing an overview of self-esteem, including the benefits of healthy self-esteem and the costs of low self-esteem. Next, we differentiate between secure high self-esteem and fragile high self-esteem, and argue that individuals high in dispositional mindfulness may have greater capacity for secure high selfesteem. We then review evidence from clinical and experimental studies examining causal associations between mindfulness and self-esteem. Finally, we conclude with a discussion of how mindfulness-based interventions may enhance healthy self-esteem and outline directions for future research.

\section{Self-Esteem}

Self-esteem refers to an individual's evaluation of his or her own self-worth, and it is considered to be a relatively stable personality trait that varies across individuals (Kernis, 2003; Waterman, 1992). Self-esteem is generally considered a fundamental human desire, and individuals are particularly motivated to maintain high self-esteem and engage in efforts to protect their self-esteem when it is threatened. Two main theories have been proposed to explain the human need for self-esteem; namely, sociometer theory (Leary, 1999; Leary, Tambor, Terdal \& Downs, 1995) and terror management theory (Greenberg, Pyszczynski, \& Solomon, 1986). According to the sociometer theory of self-esteem (Leary, 1999; Leary et al., 1995), self-esteem is an evolved system that serves as a meter of social acceptance and relationship quality with others. From an evolutionary perspective, humans are driven to maintain connection with others, and strive toward acceptance from peer groups. Thus, 
individuals are not necessarily motivated to maintain high self-esteem as the end goal, but rather to maintain acceptance from other people. This then motivates behaviours designed to enhance the chances of being valued and accepted by others. Consistent with this proposition, Leary and colleagues (1995) found that individuals' self-esteem correlates with the extent to which they believe they are accepted or excluded, with self-esteem decreasing in response to social exclusion.

Terror Management Theory (TMT) offers a distinct though somewhat related explanation as to why humans desire self-esteem (Greenberg et al., 1992; Greenberg et al., 1986; Pyszczynski et al., 2004). Humans are in the unique position of being aware of their own mortality and the inevitability of death. This awareness generates significant existential anxiety, and motivates individuals to engage in anxiety buffering defensive strategies. TMT posits that humans are motivated to pursue self-esteem as it serves as a buffer to existential death anxiety. Specifically, self-esteem is derived from meeting the standards and expectations of one's particular culture, and provides individuals with a sense that they are a valuable contributor to the world, and thus their life has meaning and importance (Pyszczynski et al., 2004). Consistent with the TMT perspective, experimental studies indicate that high self-esteem buffers death anxiety, and that priming mortality leads individuals to engage in efforts to increase their self-esteem. However, when individuals are experimentally convinced of the existence of an afterlife, their efforts to increase self-esteem in response to mortality salience primes are diminished (Pyszczynski et al., 2004). In brief, self-esteem is a fundamental human desire, and individuals are particularly motivated to maintain high self-esteem, and engage in efforts to protect self-esteem when it comes under threat.

Self-esteem is an important construct, and is related to a variety of positive psychological and social outcomes, including general psychological adjustment, positive 
emotion, social confidence, prosocial behaviour, work well-being, satisfying relationships, and overall life satisfaction (Diener, Emmons, Larsen \& Griffin, 1985; Leary \& MacDonald, 2003; Orth \& Robins, 2014). In addition, a meta-analysis of 77 longitudinal studies suggested that low self-esteem predicts depression; low self-esteem is not simply an outcome of depression (Sowislow \& Orth, 2013). Finally, low self-esteem in adolescence prospectively predicts poorer mental and physical health and higher criminal behaviour in adulthood, compared to those with high self-esteem (Trzesniewski et al., 2006). In brief, much evidence indicates that high self-esteem is a positive personal resource.

Despite the pervasive pursuit for high self-esteem, and the widely documented positive outcomes associated with high self-esteem, several researchers have noted that high self-esteem is not always beneficial (e.g., Deci \& Ryan, 1995; Jordan, Spencer, Zanna, Hoshino-Browne \& Correll, 2003; Kernis, 2003; Ryan \& Brown, 2003). For example, some studies have found that high self-esteem is associated with increased prejudice (Verkuyten \& Masson, 1995), defensive self-enhancement (Baumeister, Heatherton \& Tice, 1993) and heightened violence and aggression (Baumester, Smart \& Boden, 1996). What might explain these somewhat counterintuitive findings? One possibility is that there are two forms of high self-esteem; one associated with positive outcomes and one with negative outcomes. Accordingly, several researchers have proposed that high self-esteem can indeed take two forms (Ryan \& Brown, 2003; Kernis, 2003); namely, secure high self-esteem and fragile high self-esteem.

Secure high self-esteem refers to a positive self-view that is grounded in reality and is not easily threatened, whereas fragile high self-esteem reflects a positive self-view and feelings of self-worth that require frequent validation, are vulnerable to threat, and oftentimes reflect some form of self-deception or compensation for underlying feelings of low selfesteem (Zeigler-Hill, 2006). There are several ways in which secure and fragile high self- 
esteem have been conceptualised and distinguished. For example, defensive high self-esteem (Jordan et al., 2003; Kernis, 2003) refers to a defensive attempt to bolster or enhance explicit views of oneself, despite implicit or unconscious low self-esteem. Similarly, contingent high self-esteem is an additional conceptualisation of fragile high self-esteem. Contingent high self-esteem refers to the tendency to base one's self-worth and self-value on achievements, or meeting an ideal standard relative to others. Deci and Ryan (1995) note that when these achievements or standards on which this self-esteem is contingent upon cease, self-esteem also tends to decrease.

In summary, several terms have been used in the literature to differentiate between secure forms of self-esteem and fragile high self-esteem (i.e., defensive high self-esteem, contingent high self-esteem). Although there are differences between the various conceptualisations of self-esteem, for the purposes of the present review we use the terms secure high self-esteem and fragile high self-esteem throughout the chapter, and refer to the more specific forms of fragile high self-esteem, namely defensive high self-esteem and contingent high self-esteem, only when reporting on individual studies using these specific conceptualisations. We propose here that mindfulness is likely to be associated with high self-esteem and, in particular, may facilitate the development of secure high self-esteem as opposed to fragile high self-esteem.

\section{Dispositional Mindfulness and Self-Esteem}

In the present chapter we use the definition of mindfulness proposed by Kabat-Zinn whereby mindfulness is defined as "paying attention in a particular way: on purpose, in the present moment, non-judgmentally” (Kabat-Zinn, 1994, p. 4). Consistent with the definition outlined by Kabat-Zinn (1994), Bishop et al. (2004) propose that mindfulness includes two core components: (1) the self-regulation of attention, and, (2) a certain orientation to experience. 
Self-regulation of attention refers to the process of directing attention towards the present moment, coupled with the ability to observe and attend to the ever-changing stream of thoughts, emotions and sensations that individuals experience at any moment. Bishop et al. (2004) argue that self-regulation of attention facilitates pure awareness of thoughts, feelings and sensations based on direct experience rather than elaborative processing of these experiences or becoming involved in rumination about these experiences. The second component of the conceptualisation outlined by Bishop et al. (2004) is a certain orientation to experiences, which refers to a non-judgmental and curious stance directed towards experiences in the present moment, together with an attitude of openness and acceptance to whatever may arise in each moment.

There have been a number of measures of mindfulness developed (e.g., Baer, Smith, Hopkins, Krietemeyer \& Toney, 2006; Brown \& Ryan, 2003; Lau et al., 2006; Walach, Buchheld, Buttenmuller, Kleinknecht \& Schmidt, 2006). To provide a more in depth analysis of the construct of mindfulness, Baer et al., (2006) examined the underlying factor structure assessed by items from five measures of mindfulness and found a five-factor solution. The factors identified were: 'observing', which refers to the process of noticing internal and external experiences, including thoughts, physical sensations, and emotions; 'non-judging of inner experiences', which refers to taking a non-judgemental and accepting stance to internal experiences such as difficult thoughts, emotions, and physical sensations; 'describing' which is the ability to label with words one's own internal experiences; 'non-reactivity' to inner experiences refers to the capacity to remain present with thoughts and emotions without impulsively responding to them, and without being either consumed by them or seeking to avoid these experiences; finally, 'acting with awareness' refers to the process of paying attention to the present moment rather than being preoccupied with thoughts or emotions about the future or past (Baer et al., 2006; Baer et al., 2008). The five facets are moderately 
correlated with each other, but differentially predict theoretically related outcomes. With regards to the association between mindfulness and self-esteem, identification of the relative importance of a range of facets of mindfulness in the prediction of self-esteem may be a useful focus with important clinical implications.

Why might high dispositional mindfulness enhance self-esteem? We have argued previously (Pepping et al., 2013) that individuals dispositionally high in mindfulness may be less consumed by the thoughts and emotions that characterize low self-esteem, and thus have greater capacity to cope with these experiences. A key feature of mindfulness is a decentered stance towards thoughts. Further, individuals higher in mindfulness tend to possess a nonjudgmental, open and receptive stance to their experiences, and their thoughts and emotions (Baer et al., 2006), which may allow them to be less consumed by harsh, critical, and judgmental thoughts relating to the self. Although it may be that those high in mindfulness are less likely to generate harsh negative thoughts about the self in the first place, it is also likely that high mindfulness enables individuals to respond to harsh and self-critical thoughts, when they do occur, more adaptively.

Drawing from Ryan et al.’s (2007) discussion of attachment and mindfulness, individuals who have low self-esteem have cognitive biases that are based on past experiences, and deeply held beliefs about the self that are oftentimes highly negative. However, mindfulness may allow an individual to transcend these cognitive biases and maladaptive schemas. Mindfulness facilitates non-judgmental acceptance of the present moment, including thoughts and emotions, without being influenced or overwhelmed by cognitive biases relating to the past. Therefore, high dispositional mindfulness may act as a buffer to low self-esteem. Individuals who are high in dispositional mindfulness may have enhanced capacity to step back from, and cope with, negative thoughts relating to the self. 
Consistent with the proposition that high dispositional mindfulness should be related to increased self-esteem, several studies have found a positive relationship between mindfulness and self-esteem (Brown \& Ryan, 2003; Michalak, Teismann, Heidenreich, Strohle \& Vocks, 2011; Rajamaki, 2011; Rasmussen \& Pidgeon, 2011; Thompson \& Waltz, 2008). Very little research has examined which specific facets of mindfulness predict increased self-esteem. Michalak et al. (2011) found that 'acceptance' (similar to non-judging of experiences) predicted increased mindfulness. However, the research focussed only on the acceptance facet, and the other facets of mindfulness were therefore not examined. To extend prior research examining the association between mindfulness and self-esteem, we recently conducted two studies examining the association between mindfulness and self-esteem (Pepping, O’Donovan \& Davis, 2013). The research had two major aims; the first was to examine associations between individual facets of mindfulness and self-esteem (Study 1). The second focus was to examine the effects of a brief experimental mindfulness induction on self-esteem (Study 2).

With regards to the association between specific facets of mindfulness and selfesteem, we argued that understanding the relative importance of various facets of mindfulness in the prediction of self-esteem may inform mindfulness-based clinical interventions to increase self-esteem. That is, understanding the specific components of mindfulness that relate to increased self-esteem would enable clinicians to tailor interventions, focusing in particular on the specific processes that predict self-esteem.

We predicted that four of the five mindfulness facets (non-reactivity, awareness, labeling, and non-judging of inner experience) would significantly predict self-esteem (Pepping et al., 2013, Study 1) as measured by the Rosenberg self-esteem scale (Rosenberg, 1965). Consistent with predictions, individuals scoring high on these four facets of mindfulness were higher in self-esteem. We argued that 'non-judging' should facilitate 
increased self-esteem as individuals higher in this facet may be better able to take a nonjudgmental, and less self-critical stance towards themselves. In addition, they may be less prone to judging and evaluating specific thoughts themselves (Baer et al., 2006, 2008). That is, 'non-judging' facilitates a non-judgmental stance towards thoughts, emotions, and experiences, and these experiences are observed from a neutral, objective standpoint, as opposed to being judged as good or bad (Baer et al., 2006). With regards to self-esteem, those who are higher on the non-judging facet may be less likely to experience self-critical thoughts in the first place, but may also display enhanced capacity to perceive these thoughts purely as thoughts, rather than evaluating them or becoming caught up in the content. Interestingly, 'non-judging' was most strongly associated with increased self-esteem which suggests that the ability to maintain an open and non-judgmental approach to thoughts, feelings and sensations has a particularly positive effect on self-esteem. Individuals high on this facet may be less likely to get caught up in harsh judgmental thoughts about the self.

The 'labeling' subscale was also associated with higher self-esteem. Individuals with greater capacity to label, describe, and express cognitive and emotional experiences may be less likely to become mindlessly caught up in self-critical thoughts about themselves (Baer et al., 2006). Specifically, self-critical thoughts can be labeled, which we proposed might allow the individual to continue with other activities without becoming overwhelmed by these experiences. This may be why psychotherapies such as Dialectical Behaviour Therapy (DBT) encourage individuals to label and describe thoughts and emotions (Linehan, 1993). In brief, the ability to 'label' thoughts and feelings with words appears to be associated with increased self-esteem, perhaps reflecting the beneficial effects of being able to identify self-critical thoughts. 
Individuals high on the 'non-reactivity' facet were also higher in self-esteem. It may be that these individuals are able to allow internal experiences such as thoughts and feelings relating to the self to enter and leave awareness, without engaging in efforts to avoid or get rid of these experiences, but also without engaging in rumination (Baer et al., 2006, 2008). Rather than defensively trying to bolster self-esteem or reduce self-critical thoughts, individuals high on the 'non-reactivity' facet may be better able to experience these thoughts and emotions without responding in maladaptive ways. It seems likely that 'non-reactivity' enables individuals to treat harsh and self-critical thoughts associated simply as thoughts, rather than a true reflection of reality.

We also predicted that the 'acting with awareness' subscale would be associated with increased self-esteem, as individuals high on this facet are able to attend to the presentmoment as opposed to being distracted by self-critical thoughts. Low 'awareness', on the other hand, is associated with the tendency to get caught up in thoughts about the future or past. In line with this proposition, we found that individuals higher on the 'acting with awareness' subscales were higher in self-esteem, suggesting that the capacity to maintain present-focussed attention may assist with an individual's ability to transcend deep-seated negative beliefs relating to the self.

The 'observe' facet did not predict self-esteem. It thus appears that noticing or observing self-critical thoughts and difficult emotions does not necessarily enhance selfesteem, but rather it is the attitude or stance taken towards these experiences that is important. It is probable that individuals with both high and low self-esteem may observe self-critical thoughts. However, it appears that the 'relationship' one has with these experiences is more strongly related to increased self-esteem. The finding that 'observe' did not predict increased self-esteem is consistent with this proposition. 
The study described here (Pepping et al., 2013, Study 1) represented the first attempt to examine the 'relative' importance of five facets of mindfulness in the prediction of healthy self-esteem. This level of analysis allows us to understand more specifically which aspects of mindfulness are associated with self-esteem. It appears that present-focussed attention, a non-judgmental, non-reactive, and accepting stance towards thoughts and emotions, and the ability to label thoughts and feelings all contribute to the development of healthy self-esteem. It is important to note, however, that the research reviewed above, although very useful with regards to providing insight into the specific factors of mindfulness relating to increased selfesteem, is cross-sectional. This limits the extent to which we can conclude that mindfulness actively enhances self-esteem. We therefore turn to the use of mindfulness-based clinical interventions and experimental studies examining causal associations between mindfulness and self-esteem.

\section{Mindfulness-Based Interventions to Cultivate Healthy Self-Esteem}

Mindfulness-based clinical interventions

As mentioned previously, past research has demonstrated that mindfulness is associated with high self-esteem (Brown \& Ryan, 2003; Michalak et al., 2011; Rajamaki, 2011; Rasmussen \& Pidgeon, 2011; Thompson \& Waltz, 2008), and that several specific facets of mindfulness each make unique contributions in the prediction of high self-esteem (Pepping et al., 2013, Study 1). To more fully understand the clinical applications of mindfulness for cultivating healthy self-esteem, however, it is necessary to examine the effects of mindfulness-based clinical interventions on self-esteem.

Several studies have shown that mindfulness-based clinical interventions have beneficial effects on self-esteem. Goldin and Gross (2010) and Goldin, Ramel, and Gross (2009) examined the efficacy of an eight week mindfulness-based stress reduction 
intervention for the treatment of social anxiety disorder. The researchers also included measures of self-esteem. Consistent with the mindfulness-based stress reduction protocol, participants completed eight 2.5-hour sessions, and a half-day retreat. Further, daily practice of mindfulness skills between sessions was encouraged. Participants who completed the intervention displayed improvements not only in their symptoms of social anxiety, but also displayed increased self-esteem. It is important to note, however, that the study did not utilise a control group which does limit the extent to which results can be attributed specifically to the intervention.

In a mindfulness-based stress reduction intervention for adolescents with depression, Biegel, Brown, Shapiro, and Schubert (2009) found that participants in the mindfulness intervention condition displayed a reduction in depression, and an increase in self-esteem, relative to a treatment as usual control condition. Participants in the mindfulness intervention group received eight weekly two hour group sessions of mindfulness that emphasised both formal mindfulness meditation training and informal mindfulness practice.

More recently, Rajamaki (2011) examined the effects of mindfulness-based stress reduction on different forms of self-esteem. Specifically, the authors examined whether an eight-week mindfulness intervention would increase basic self-esteem, and reduce competence based self-esteem, which is a more fragile form of self-esteem, characterised by the tendency to strive for achievement in order to counteract or compensate for underlying low self-esteem (Johnson \& Blom, 2007). Interestingly, participants reported decreased competence based self-esteem, and increased basic self-esteem from pre- to post-intervention. Also of interest, increased mindfulness resulting from the intervention predicted decreased competence based self-esteem. However, increased mindfulness across the intervention did not predict increased basic self-esteem. Although it is important to note that there was no control condition included in this study, results do suggest that enhancing mindfulness may 
facilitate the development of secure forms of self-esteem, and reduce more fragile, insecure forms of self-esteem.

Results of the abovementioned studies clearly suggest that mindfulness-based interventions may be beneficial for cultivating self-esteem in a range of populations, including adolescents with depression (Biegel et al., 2009) and individuals with social anxiety disorder (Goldin et al., 2009; Goldin et al., 2010). Interestingly, enhancing mindfulness is also associated with a decrease in defensive forms of self-esteem (i.e., competence-based self-esteem) and an increase in basic self-esteem (Rajamaki, 2011). This is consistent with the proposition that mindfulness may facilitate the development of healthy, secure forms of self-esteem, as opposed to defensive or fragile high self-esteem, an issue that will be discussed in more depth below. We now turn to the use of experimental mindfulness inductions to examine causal associations between mindfulness and self-esteem.

Experimental mindfulness inductions

The results of the mindfulness-based clinical interventions reported above clearly demonstrate that mindfulness based interventions lead to improved self-esteem. However, it is somewhat difficult to draw definitive conclusions here because of the nature of the interventions, the lack of control groups in some studies, and the nature of the samples used in the studies. It is unclear whether mindfulness itself was directly responsible for the improved self-esteem, or whether other factors associated with clinical interventions, such as the therapeutic relationship, group cohesion, or the alleviation of suffering associated with the presenting problem itself (i.e., depression and social anxiety) were more important in enhancing self-esteem. To examine direct, immediate causal associations between mindfulness and psychological outcomes, researchers are beginning to use brief experimental mindfulness inductions (e.g., Arch \& Craske, 2006; Eifert \& Heffner, 2003; Keng et al., 
2011). Researchers have examined the effects of experimental mindfulness inductions on dysphoric mood (Broderick, 2005), negative affect and emotional volatility (Arch \& Craske, 2006), symptoms of panic attacks (Eifert \& Heffner, 2003) self-reported physical pain (Braams, Blechert, Boden \& Gross, 2012) and pain tolerance (Liu, Wang, Chang, Chen \& Si, 2013).

We recently extended this research to investigate whether a brief mindfulness induction could lead to increased state self-esteem (Pepping et al., 2013, Study 2). We proposed that participants in the experimental mindfulness induction condition should increase not only in state mindfulness as a result of the mindfulness induction, but also should display an increase in state self-esteem. No such effects were predicted for those in the control condition. Here we briefly describe the experimental procedures used in the study, and outline the findings.

Sixty-eight participants were randomly assigned to either a 15-minute mindfulness induction condition or to a control condition. Participants in the mindfulness condition completed a mindfulness meditation of the breath that also included a focus on mindfulness of thoughts. The meditation script was read aloud to participants in groups of up to 10 participants per session. The script is included in Appendix A of this chapter. Participants in the control condition were read a 15-minute story about Venus Fly Trap plants. This control condition was chosen because it was unlikely to enhance mindfulness or self-esteem, but was still an active condition whereby participants were required to listen to a story. All participants completed brief measures of state mindfulness and state self-esteem before and after the experimental manipulation.

Results revealed that state mindfulness significantly increased in the experimental condition (pre-post) and not in the control condition, which indicated that the manipulation (inducing a mindful state) was successful. With regards to whether inducing mindfulness led 
to change in self-esteem, we found that state self-esteem also increased in the experimental condition (pre-post) and not in the control condition, demonstrating that enhancing state mindfulness led to a positive change in state self-esteem. Although this experimental mindfulness induction was successful in enhancing both state mindfulness and state selfesteem, it is important to consider that the effects of this brief mindfulness induction are likely to be temporary. However, the findings are consistent with the cross-sectional evidence of a relationship between mindfulness and self-esteem, and are also consistent with evidence from clinical studies that demonstrate that enhancing mindfulness leads to increased selfesteem. Importantly, results reported by Pepping et al. (2013, Study 2) demonstrate that mindfulness has a 'direct' positive influence on self-esteem. In brief, systematically enhancing mindfulness leads to positive effects on self-esteem.

\section{Mindfulness to Cultivate Secure as Opposed to Fragile High Self-Esteem}

We propose that mindfulness should cultivate secure rather than fragile high self-esteem. Mindfulness emphasizes the importance of a non-judgmental and accepting stance to difficult thoughts and emotions that arise moment to moment (Baer, 2003). In the context of selfesteem, an individual high in dispositional mindfulness would therefore notice positive and negative thoughts about the self without getting 'caught up' in these experiences and engaging in elaborative processing, but also without engaging in efforts to avoid or change these experiences. This is consistent with the finding that individuals high in four facets of mindfulness, each of which involve mindful acceptance and awareness (non-reactivity, labelling, non-judging, and awareness) were higher in self-esteem (Pepping et al., 2013, Study 1). 
Importantly, the processes of mindful acceptance and awareness are vastly different from the cognitive strategy of reappraisal, which refers to the process of cognitively reappraising the situation by changing the way one views the situation (Gross, 2011; Gross \& John, 2003). Cognitive reappraisal is somewhat removed from, and even antithetical to, the concept of mindfulness (Chambers, Gullone \& Allen, 2009). Indeed, Chambers and colleagues (2009) argue that reappraisal may even reflect a form of experiential avoidance at its most extreme. Much evidence indicates that efforts to avoid or control difficult thoughts and emotions can have paradoxical effects by increasing or intensifying these experiences (e.g., Hayes, Luoma, Bond, Masuda, \& Lillis, 2006; Salters-Pedneault, Tull, \& Roemer, 2004; Wenzlaff \& Wegner, 2000). Engaging in efforts to cognitively reappraise and bolster explicit views of oneself despite underlying low levels of self-esteem may represent defensive high self-esteem (a form of fragile high self-esteem; Jordan et al., 2003; Kernis, 2003). Mindfulness, on the other hand, allows an individual to accept and transcend these thoughts relating to the self without reacting to them.

Similarly, reappraisal could lead to contingent high self-esteem, which is an additional conceptualisation of fragile high self-esteem (Deci \& Ryan, 1995). Contingent high self-esteem refers to the process of temporarily bolstering one's self view based on achievements, successes, or adherence to some ideal standard (Deci \& Ryan, 1995). However, as noted earlier, when the standards, achievements or successes on which this form of self-esteem is contingent upon are threatened or cease, self-esteem is likely to suffer as a result. We therefore propose that strategies used to cognitively reappraise and temporarily bolster views of the self may not lead to secure forms of self-esteem, whereas mindfulness may facilitate the ability to non-judgmentally accept difficult thoughts and emotions without buying into them, and without reacting to them impulsively. 
Perhaps the most compelling evidence that mindfulness may lead to secure forms of self-esteem comes from our recent experimental study described earlier whereby participants were induced with a mindful state, which resulted in increased state self-esteem (Pepping et al., 2013, Study 2). Interestingly, this experimental mindfulness induction did not explicitly target self-esteem. Participants in the mindfulness meditation condition were not asked to think more positively about themselves, no attempt was made to temporarily bolster selfesteem, and participants were also not asked to think about achievement or positive aspects of their lives. Instead, the focus of the induction (consistent with mindfulness) was to adopt a different 'relationship' to thoughts and feelings (Kabat-Zinn, 1994; Baer et al., 2006). It appears that participants in the mindfulness induction condition were better able to let go of negative thoughts about the self, and were more open to perceiving thoughts purely as events in the mind, rather than a true reflection of reality. The above description may reflect a form of secure high self-esteem as opposed to fragile high self-esteem.

Niemiec et al. (2010) recently reported on research pertaining to mindfulness and selfesteem from a terror management perspective. As mentioned earlier, according to Terror Management Theory, humans attempt to regulate death anxiety by striving for self-esteem (Greenberg et al., 1992; Greenberg et al., 1986; Pyszczynski et al., 2004). Niemiec et al. (2010) found that individuals who were low in mindfulness were more likely to strive for self-esteem in response to experimental mortality salience inductions, which reflects an attempt to buffer death anxiety by bolstering self-esteem. Interestingly, the need to defensively bolster self-esteem was not observed in those high in mindfulness following the experimental mindfulness induction. Again, this is consistent with the notion that mindfulness is associated with enhanced self-esteem and, importantly, secure high selfesteem. 
In summary, both cross-sectional and experimental research suggests that mindfulness may facilitate the development of secure high self-esteem as opposed to fragile forms of selfesteem. The finding that measures of mindfulness, which focus on non-judgmental acceptance as opposed to cognitive reappraisal, predict increased self-esteem is consistent with this proposition (Brown \& Ryan, 2003; Michalak et al., 2011; Rajamaki, 2011; Rasmussen \& Pidgeon, 2011; Thompson \& Waltz, 2008). Further, experimentally inducing a mindful state, without bolstering self-esteem or restructuring self-views, led to an increase in state self-esteem (Pepping et al., 2013, Study 2), again, consistent with a non-defensive, secure form of self-esteem resulting from increased mindfulness. Mindfulness may thus be a useful way to address the underlying processes associated with low self-esteem, without temporarily bolstering positive views of oneself by focussing on achievement or other transient factors.

\section{Conclusions and Future Directions}

The findings from the research reviewed in this chapter suggest that individuals high in dispositional mindfulness tend to be high in self-esteem, and that mindfulness-based clinical interventions may assist in cultivating secure self-esteem. With regards to which specific components of mindfulness enhance self-esteem, results by Pepping et al. (2013, Study 1) indicate that the 'non-judging' facet of mindfulness most strongly relates to high self-esteem, suggesting that mindfulness-based interventions that utilize strategies to foster a nonjudgmental stance towards the self, thoughts, and emotions, may be particularly beneficial to individuals low in self-esteem. Further, 'non-reactivity', 'acting with awareness', and 'describing' were also associated with high self-esteem, again, suggesting that mindfulness strategies focussing on these specific skills may also enhance self-esteem. Importantly, the proposition that mindfulness-based clinical interventions increase self-esteem has been 
supported by both intervention studies (Biegel et al., 2009; Goldin et al., 2009; Goldin et al., 2010; Rajamaki, 2011) and experimental research (Pepping et al., 2013, Study 2).

Although this research is promising, the challenge for future research is to examine whether mindfulness-based interventions lead to enhanced self-esteem in samples specifically seeking assistance for low self-esteem, and also in non-clinical populations. It would also be very useful for future research to explore potential mediators of the relationship between mindfulness and self-esteem, and to examine the underlying mechanisms of the beneficial effects of mindfulness interventions on self-esteem. This would have particularly important clinical implications as it would elucidate the specific underlying processes responsible for any benefit from mindfulness interventions. In summary, the research reviewed in this chapter clearly demonstrates that mindfulness and self-esteem are related, that enhancing mindfulness increases self-esteem, and that specific components of mindfulness may be particularly important for cultivating self-esteem. 


\section{References}

Arch, J. J. \& Craske, M. G. (2006). Mechanisms of mindfulness: Emotion regulation following a focused breathing induction. Behaviour Research and Therapy, 44(12), 1849-1858. doi: 10.1016/j.brat.2005.12.007

Baer, R. A. (2003). Mindfulness training as a clinical intervention: A conceptual and empirical review. Clinical Psychology: Science and Practice, 10, 125-143. doi:10.1093/clipsy/bpg015.

Baer, R. A., Smith, G. T., Hopkins, J., Krietemeyer, J., \& Toney, L. (2006). Using self-report assessment methods to explore facets of mindfulness. Assessment, 13, 27-45. doi:10.1177/1073191105283504

Baer, R. A., Smith, G. T., Lykins, E., Button, D., Krietemeyer, J., Sauer, S., Walsh, E., Duggan, D., \& Williams, J. M. G. (2008). Construct validity of the five facet mindfulness questionnaire in meditating and nonmeditating samples. Assessment, 15(3), 329-342. doi: 10.1177/1073191107313003.

Baumeister, R. F., Heatherton, T. F., \& Tice, D. M. (1993). When ego threats lead to selfregulation failure: negative consequences of high self-esteem. Journal of Personality and Social Psychology, 64(1), 141-156, doi: 10.1037/0022-3514.64.1.141.

Baumeister, R. F., Smart, L., \& Boden, J. M. (1996). Relation of threatened egotism to violence and aggression: the dark side of high self-esteem. Psychological review, 103(1), 5-33, doi: 10.1037/0033-295X.103.1.5.

Biegel, G. M., Brown, K. W., Shapiro, S. L. \& Schubert, C. M. (2009). Mindfulness-based stress reduction for the treatment of adolescent psychiatric outpatients: A randomized clinical trial. Journal of Consulting and Clinical Psychology, 77(5), 855-866. doi: 10.1037/a0016241 
Bishop, S. R., Lau, M., Shapiro, S., Carlson, L., Anderson, N. D., Carmody, J., ... Devins, G. (2004). Mindfulness: A proposed operational definition. Clinical Psychology: Science and Practice, 11(3), 230-241, doi:10.1093/clipsy.bph077.

Braams, B. R., Blechert, J., Boden, M. T., \& Gross, J. J. (2012). The effects of acceptance and suppression on anticipation and receipt of painful stimulation. Journal of Behavior Therapy and Experimental Psychiatry, 43(4), 1014-1018. doi: 10.1016/j.jbtep.2012.04.001

Broderick, P. C. (2005). Mindfulness and Coping with Dysphoric Mood: Contrasts with Rumination and Distraction. Cognitive Therapy and Research, 29(5), 501-510. doi: $10.1007 / \mathrm{s} 10608-005-3888-0$

Brown, K. W. \& Ryan, R. M. (2003). The benefits of being present: Mindfulness and its role in psychological well-being. Journal of Personality and Social Psychology, 84(4), 822-848. doi: 10.1037/0022-3514.84.4.822.

Chambers, R., Gullone, E., \& Allen, N. B. (2009). Mindful emotion regulation: An integrative review. Clinical Psychology Review, 29(6), 560-572, doi: 10.1016/j.cpr.2009.06.005.

Deci, E. L., \& Ryan, R. M. (1995). Human autonomy: The basis for true self-esteem. In M. Kernis (Ed.), Efficacy, agency, and self-esteem (pp.31-49). New York: Plenum.

Diener, E., Emmons, R. A., Larsen, R. J. \& Griffin, S. (1985). The satisfaction with life scale. Journal of Personality Assessment, 49(1), 71-75. doi:10.1207/s15327752jpa4901_13

Eifert, G. H., \& Heffner, M. (2003). The effects of acceptance versus control contexts on avoidance of panic-related symptoms. Journal of Behavior Therapy and Experimental Psychiatry, 34, 293-312, doi: 10.1016/j.jbtep.2003.11.001. 
Goldin, P. R., \& Gross, J. J. (2010). Effects of mindfulness-based stress reduction (MBSR) on emotion regulation in social anxiety disorder. Emotion, 10(1), 83-91, doi: $10.1037 / \mathrm{a} 0018441$

Goldin, P. Ramel, W., \& Gross, J. (2009). Mindfulness meditation training and selfreferential processing in social anxiety disorder: Behavioral and neural effects. Journal of Cognitive Psychotherapy: An International Quarterly, 23(3), 242-257, doi: 10.1891/0889-8391.23.3.242.

Greenberg, J., Pyszczynski, T., \& Solomon, S. (1986). The causes and consequences of a need for self-esteem: A terror management theory. In Public Self and Private Self (pp. 189-212). Springer: New York.

Greenberg, J., Solomon, S., Pyszczynski, T., Rosenblatt, A., Burling, J., Lyon, D., ... \& Pinel, E. (1992). Why do people need self-esteem? Converging evidence that selfesteem serves an anxiety-buffering function. Journal of personality and social psychology, 63(6), 913-922, doi: 10.1037/0022-3514.63.6.913.

Gross, J. J. (Ed.). (2011). Handbook of Emotion Regulation. New York: Guilford Press.

Gross, J. J., \& John, O. P. (2003). Individual differences in two emotion regulation processes: implications for affect, relationships, and well-being. Journal of Personality and Social Psychology, 85(2), 348-362, doi: 10.1037/0022-3514.85.2.348.

Hayes, S.C., Luoma, J.B., Bond, F.W., Masuda, A. and Lillis, J. (2006). Acceptance and commitment therapy: Model, process and outcomes. Behaviour Research and Therapy, 44(1), 1-25. doi:10.1016/j.brat.2005.06.006.

Johnson, M., \& Blom, V. (2007). Development and validation of two measures of contingent self-esteem. Individual Differences Research, 5(4), 300-328. 
Jordan, C. H., Spencer, S. J., Zanna, M. P., Hoshino-Browne, E., \& Correll, J. Secure and defensive high self-esteem. Journal of Personality and Social Psychology, 85(5), 969-978. doi: 10.1037/0022-3514.85.5.969.

Kabat-Zinn, J. (1994). Wherever you go, there you are: Mindfulness meditation in everyday life. New York: Hyperion.

Keng, S-L., Smoski, M. J., \& Robins, C. J. (2011). Effects of mindfulness on psychological health: A review of empirical studies. Clinical Psychology Review, 31, 1041-1056, doi:10.1016/j.cpr.2011.04.006.

Kernis, M. H. (2003). Toward a conceptualization of optimal self-esteem. Psychological Inquiry, 14(1), doi: 10.1207/S15327965PLI1401_01.

Lau, M. A., Bishop, S. R., Segal, Z. V., Buis, T., Anderson, N. D., Carlson, L., ... \& Carmody, J. (2006). The toronto mindfulness scale: Development and validation. Journal of Clinical Psychology, 62(12), 1445-1467. doi: 10.1002/jclp.20326.

Leary, M. R. (1999). Making sense of self-esteem. Current directions in psychological science, 8(1), 32-35, doi: 10.1111/1467-8721.00008.

Leary, M.R., \& MacDonald, G. (2003). Individual differences in self-esteem: A review and theoretical integration. In M.R. Leary \& J.P. Tangney (Eds.), Handbook of self and identity (pp. 401-418). New York: Guilford Press.

Leary, M. R., Tambor, E. S., Terdal, S. K., \& Downs, D. L. (1995). Self-esteem as an interpersonal monitor: The sociometer hypothesis. Journal of Personality and Social Psychology, 68(3), 518-530, doi: 10.1037/0022-3514.68.3.518.

Linehan, M. M. (1993). Cognitive-behavioral treatment of borderline personality disorder. New York: Guilford Press. 
Liu, X., Wang, S., Chang, S., Chen, W., \& Si, M. (2013). Effect of a brief mindfulness intervention on tolerance and distress of pain induced by cold-pressor task. Stress and Health, 29(3), 199-204, doi: 1002/smi.2446.

Michalak, J., Teismann, T., Heidenreich, T., Strohle, G., \& Vocks, S. (2011). Buffering low self-esteem: The effect of mindful acceptance on the relationship between self-esteem and depression. Personality and Individual Differences, 50, 751-754, doi: 10.1016/j.paid.2010.11.029.

Niemiec, C.P., Brown, K.W., Kashdan, T.B., Cozzolino, P.J., Breen, W.E., Levesque-Bristol, C., \& Ryan, R.M. (2010). Being present in the face of existential threat: The role of trait mindfulness in reducing defensive responses to mortality salience. Journal of Personality and Social Psychology, 99(2), 344-365, doi: 10.1037/a0019388.

Orth, U., \& Robins, R. W. (2014). The Development of Self-Esteem. Current Directions in Psychological Science, 23(5), 381-387, doi: 10.1177/0963721414547414.

Pepping, C. A., O’Donovan, A. \& Davis, P. (2013).The positive effects of mindfulness on self-esteem. The Journal of Positive Psychology, 8(5),376-386, doi:10.1080/17439760.2013.807353.

Pyszczynski, T., Greenberg, J., Solomon, S., Arndt, J., \& Schimel, J. (2004). Why do people need self-esteem? A theoretical and empirical review. Psychological bulletin, 130(3), 435-468, doi: 10.1037/0033-2909.130.3.435.

Rajamaki, S. (2011). Mindfulness-based stress reduction: Does mindfulness training affect competence based self-esteem and burnout? Unpublished Masters Dissertation. Stockholm University.

Ryan, R. M., \& Brown, K. W. (2003). Why we don't need self-esteem: On fundamental needs, contingent love, and mindfulness. Psychological Inquiry, 14(1), 71-76. 
Ryan, R. M., Brown, K. W., \& Creswell, J. D. (2007). How integrative is attachment theory? Unpacking the meaning and significance of felt security. Psychological Inquiry, 18(3), 177-182. doi: 10.1080/10478400701512778.

Rasmussen, M. K. \& Pidgeon, A. M. (2011).The direct and indirect benefits of dispositional mindfulness on self-esteem and social anxiety. Anxiety, Stress \& Coping, 24(2), 227233. doi: 10.1080/10615806.2010.515681

Rosenberg, M. (1965). Society and the adolescent self-image. New Jersey: Princeton University Press.

Salters-Pedneault, K., Tull, M. T., \& Roemer, L. (2004). The role of avoidance of emotional material in the anxiety disorders. Applied and Preventative Psychology, 11(2), 95114. doi:10.1016/j.appsy.2004.09.001

Sowislo, J. F., \& Orth, U. (2013). Does low self-esteem predict depression and anxiety? A meta-analysis of longitudinal studies. Psychological Bulletin, 139(1), 213-240, doi: 10.1037/a0028931.

Thompson, B. L. \& Waltz, J. A. (2008). Mindfulness, self-esteem, and unconditional selfacceptance. Journal of Rational-Emotive Cognitive-Behavior Therapy, 26(2), 119126. doi: 10.1007/s10942-007-0059-0.

Trzesniewski, K. H., Donnellan, M. B., Moffitt, T. E., Robins, R. W., Poulton, R., \& Caspi, A. (2006). Low self-esteem during adolescence predicts poor health, criminal behavior, and limited economic prospects during adulthood. Developmental Psychology, 42(2), 381-390, doi: 10.1037/0012-1649.42.2.381.

Verkuyten, M., \& Masson, K. (1995). 'New racism', self-esteem, and ethnic relations among minority and majority youth in the Netherlands. Social Behavior and Personality: An International Journal, 23(2), 137-154, doi: 10.2224/sbp.1995.23.2.137. 
Walach, H., Buchheld, N., Buttenmuller, V., Kleinknecht, N., \& Schmidt, S. (2006).

Measuring mindfulness--The Freiburg Mindfulness Inventory (FMI). Personality and Individual Differences, 40(8), 1543-1555. doi: 10.1016/j.paid.2005.11.025

Waterman, A.S. (1992). Identity as an aspect of optimal psychological functioning. In G.R. Adams, T.P. Gullotta, \& R. Montemayor (Eds.), Adolescent identity formation (pp. 50-72). Newbury Park, CA: Sage.

Wenzlaff, R. M., \& Wegner, D. M. (2000). Thought suppression. Annual Review of Psychology, 51(1), 59-91,doi:10.1146/annurev.psych.51.1.59.

Zeigler-Hill, V. (2006). Discrepancies between implicit and explicit self-esteem: Implications for narcissism and self-esteem instability. Journal of Personality, 74(1), 119-144, doi: 10.1111/j.1467-6494.2005.00371.x. 


\section{Appendix A}

\section{Experimental Mindfulness Induction Script}

I am now going to take you through a 15 minute guided mindfulness meditation. The purpose of this meditation is not necessarily to feel more relaxed or calm or better than you did at the start of the meditation. The purpose of this is to just simply practice mindfulness. So taking a few moments now to settle into a comfortable position - wiggle into a position so that your back is straight, but not rigid. Place your feet squarely on the ground. If you wear glasses you may like to take them off. And gently close your eyes if you feel comfortable doing so. And if not, just find a spot on the floor to focus on.

Feeling all the points of contact between your body and the chair, and just settling into the stillness. Let's begin by just noticing that you can feel your feet on the ground. Notice that you can feel the bottom of your feet in your shoes. Just settling into this... bringing attention now to the feeling of the palms of your hands. And either paying attention to what you're touching or the feeling of contact. Or perhaps the feeling of the air or the temperature of the air on your palms. And just bringing all your attention and awareness to this part of the body.

And now shifting attention to the sensation of breathing. We're not trying to change the breath in any way. It doesn't have to become deeper or slower or calmer. Just paying attention to the breath as it is in this moment. Throughout this meditation we will be using the breath as an anchor. So every time you find that your mind wanders, you start thinking, or responding to sounds or thoughts as they arise, every time you notice this, just time and time again, bring your mind back to the breath - that is, your attention back to the breath. And so now for the next few moments, just sitting, and bringing your attention to the feeling of the in-breath, and the feeling of the out-breath. Holding in awareness that part of the body where 
the breath feels most vivid or strong for you. It might be your abdomen, or your chest or nose or throat. Just bringing all your attention and awareness to that part.

Every time you find your mind has wandered, just gently bring your attention back to the breath. You may already find that your mind has wandered. And your mind is just doing what minds do. You may be noticing thoughts about the meditation, whether you are doing it right, whether this is boring. You may have thoughts about how relaxing or calming this feels. No matter what your thoughts are, just know that they are thoughts, they're mental events that come into the mind, and just as easily, if you leave them well alone, they will also go out of your mind, and be replaced by more. You may be noticing bizarre or random thoughts. You might be planning what you will do for the rest of the day or tomorrow. The purpose of a mindfulness meditation is not to stop your thoughts or suppress them or resist them or get rid of them. It's just to know that you're thinking and then shift your attention back to the breath. So your thoughts become like background chatter - like a radio going in the background - they are there, your mind is chattering away, and you are just not getting caught up with it.

Just noticing your breathing and what is happening in the present moment. So just bringing your attention to where the mind is now. And if you need to come back to the breath, then gently bring your mind back. You may also like to notice the reactions when you find that your mind has wandered when you bring awareness to the focus of the thoughts. Perhaps you have a reaction that you don't want to be having this thought, or you shouldn't be having this thought at the moment. Perhaps if you are finding it difficult for your mind to settle, that it shouldn't be this way, or that this is hard. Just notice those thoughts as thoughts. Your mind giving a commentary of what it is thinking at the moment. Nothing more and nothing less. The thoughts are not necessarily true. The thoughts are not necessarily things to be believed. And the thoughts are not necessarily things to be acted upon. Just let the 
thoughts do their thing, and just bring your attention back to the breath, no matter what your thoughts are telling you.

So breathing in, and breathing out... just simply observing the breath, in this moment.... And now in this moment.... Just breathing in, and just breathing out. Being aware of everything that is happening, in each moment, as it passes. Your thoughts are going to be there whether you want them to be there or not. So sometimes you may as well just let them. But bringing your attention to the experience of breathing in, and breathing out. And letting your thoughts come and go as they will. And you may be noticing themes to your thoughts - these are the 'hard and boring' thoughts. These are the 'planning' thoughts. Just when you notice the theme of the thoughts, just remember they are just thoughts too, and bringing your attention back to the breathing.

You may be also becoming aware of feelings and sensations as you're sitting for this amount of time. You may be noticing themes of discomfort, or itches as you sit. See if you can experience these just as sensations. You may notice thoughts like this really hurts, or this is unbearable, or I have to scratch. And again, just because they're thoughts doesn't mean they are real or that you have to obey them. Just be willing to experience it - be open to allowing it to be there. Holding these sensations in one part of awareness, and focussing on the breath at the same time.

And observing your minds reaction. Perhaps your mind is irritated. Perhaps your mind is telling you to scratch, or to move. And if you do decide to move, or to itch, just do so mindfully. And then just coming back to the breath, and allowing things to be, just as they are. Just breathing in, and just breathing out. Letting thoughts and sensations just enter awareness and then leave awareness. And continue to focus on your breath. So mindfulness is awareness of everything that is happening in the present moment. Just allowing it to be there. 
Being willing to have the experience you are having. And just breathing in, and just breathing out.

Being aware of whatever is happening in the present moment. If you find you're lost in thoughts, just notice where your mind went. And bring your mind back to the breath. You might find that the background chatter gets less. Or maybe it doesn't. Regardless of what's happening... just come back to the breath.

And now bring your attention and awareness to the feeling in your body on the chair. And all the points of contact between you and the surface. And now just notice that you can feel your feet on the ground. Notice that you can feel the bottom of your feet in your shoes. Then bring your attention to the palms of your hands. Whether they are touching the chair, or your body, or whether you can just feel the temperature of the air on them...just bringing your attention to the palms of your hands. Now gently bring your attention and awareness of the room around you. And when you're ready, open your eyes, and come back to the room.

Script used in Study 2 reported by Pepping, O’Donovan and Davis (2013). 